\section{EL IDEAL DE CUERPO DELGADO Y LA FORMACIÓN DEL SUJETO NEOLIBERAL: UN ESTUDIO DE CASO}

\author{
O IDEAL DO CORPO MAGRO E A FORMAÇÃO DO SUJEITO
}

NEOLIBERAL: UM ESTUDO DE CASO C?

THE THIN BODY IDEAL AND THE CONSTITUTION OF THE

NEOLIBERAL SUBJECT: A CASE STUDY C P

doit' https://doi.org/10.22456/1982-8918.104124

Cornelio Águila*<cornelio@ual.es>

Álvaro Sicilia* <asicilia@ual.es>

Lidia Segovia*<lidya_308@hotmail.com>

*Universidad de Almería. Almería, España.
Resumen: El objetivo de este artículo es mostrar mediante un estudio de caso único, cómo la discrepancia entre la imagen corporal del ideal delgado y la real puede estimular los procesos de formación del sujeto neoliberal. El instrumento de recogida de información fue la entrevista cualitativa, abierta y semiestructurada. Los resultados muestran cómo la imagen corporal del ideal delgado puede funcionar como una tecnología de poder, al exhibir un cuerpo normalizado y homogéneo que estimula los procesos reflexivos críticos sobre uno mismo, procesos que pueden abocar en prácticas de transformación del propio cuerpo, contribuyendo a la construcción de sujetos sensibles al impulso neoliberal. Finalmente, aportamos algunas implicaciones pedagógicas para el desarrollo de tecnologías del yo liberadoras orientadas al autoconocimiento y al aumento de la consciencia crítica.

Palabras clave: Imagen corporal. Tipificación del cuerpo. Informe de caso. Concienciación.
Recibido en: 10-06-2020 Aprobado en: 25-10-2020 Publicado en: 08-12-2020

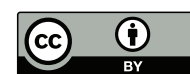

Este é um artigo publicado sob a licença Creative Commons Atribuição 4.0 Internacional (CC BY 4.0). elSSN: 1982-8918 


\section{INTRODUCCIÓN}

Las teorías socioculturales sobre la imagen corporal han destacado la influencia del discurso social, que ensalza la delgadez y critica la gordura (THOMPSON et al., 1999). Este discurso incluye modelos corporales de referencia del ideal delgado, el cual ejerce presión, especialmente sobre las mujeres, para que alcancen esa imagen esbelta y delgada (RODGERS, 2016). Si bien existen abundantes investigaciones sobre la influencia de dicho discurso y sus posibles consecuencias negativas, tales como insatisfacción corporal o trastornos alimentarios, lo cierto es que la mayoría de ellas se han realizado utilizando modelos teóricos predictivos (CAFRI et al., 2005).

Son escasos los trabajos realizados para entender el proceso subjetivo por el que las personas quedan sometidas a la presión sociocultural hacia determinados ideales de cuerpo, a pesar de que este entendimiento podría ayudar a pensar estrategias pedagógicas y prácticas de resistencia. Utilizando la estructura teórica de Michel Foucault, en este trabajo revisamos los resultados de un caso para analizar el proceso de subjetividad que conlleva el intento por alcanzar los ideales de belleza corporal en el contexto de la cultural neoliberal.

\subsection{LA INFLUENCIA SOCIOCULTURAL SOBRE LA IMAGEN CORPORAL}

En las sociedades occidentales de nuestros días las mujeres son objeto de una gran presión por conformarse al ideal delgado de cuerpo como un reflejo de la belleza femenina (THOMPSON et al., 1999; THOMPSON; SCHAEFER, 2018). La motivación para perseguir ese cuerpo ideal delgado se transmite al asociar la delgadez con la felicidad y el éxito social, así como con ciertos rasgos valorados culturalmente como el autocontrol (BORDO, 2003). Las teorías socioculturales han resaltado el proceso de internalización como un elemento clave en el proceso de trasmisión de la presión social hacia determinado ideal de cuerpo y la promoción de una insatisfacción corporal.

El proceso de internalización ha sido entendido como el grado en el que la persona cognitivamente hace suyo o se adhiere a los ideales de cuerpo socialmente definidos, de tal forma que se implica en comportamientos (e.g., a través de la alimentación y el ejercicio) para intentar alcanzar tales ideales (THOMPSON et al., 1999). A pesar de que la apariencia física del ideal delgado es reconocida como poco realista para la mayoría de los individuos, cuando la persona internaliza dicho ideal suele experimentar sentimientos de discrepancia entre su propia imagen y la ideal, que pueden provocar malestar con su propio cuerpo (RODGERS, 2016).

De acuerdo con la teoría de la discrepancia, hay tres dominios básicos del yo (HIGGINS, 1987): el yo real, que es la representación de los atributos que alguien (uno mismo u otro) cree que realmente posee; el yo ideal, que es la representación de los atributos que alguien (uno mismo u otro) desea que posea idealmente; y el yo como debería ser, que es la representación de los atributos que alguien (uno mismo u otro) cree que debería poseer. Las discrepancias entre cómo se ve una persona a sí misma y cómo le gustaría ser (yo real-ideal), pueden llevarle a sentir insatisfacción y decepción, además de sentimientos de vergüenza si cree que no ha cumplido con las 
expectativas de los demás. Las discrepancias entre cómo una persona se ve y cómo cree que debería ser (yo real-yo debería), pueden provocar estados de agitación, miedo y ansiedad, y sentimientos de culpabilidad.

Desde este punto de vista, la imagen corporal delgada refleja una apariencia ideal que, en algunos casos, puede convertirse en un imperativo moral. Así, podríamos hablar de imagen corporal real, imagen corporal ideal y de imagen corporal normativa. Medios de comunicación y redes sociales expanden un tipo de cuerpo ideal delgado, lo cual puede crear expectativas sobre la apariencia física (CARRARD; ROTHEN, RODGERS, 2020). Estos mensajes pueden verse amplificados a través de las interacciones personales con familiares, compañeros y/o iguales, lo que contribuye a la sobrevaloración de la delgadez (RODGERS, 2016). Este ideal corporal, en ciertas personas $\mathrm{u}$ organizaciones, puede reflejar también un cuerpo normativo, toda vez que funciona como referente moral de éxito y aceptación social, y viene con un discurso incorporado que señala consignas y estrategias para su consecución (DRAKE; RADFORD, 2019).

Una clave para entender las consecuencias negativas (e.g., insatisfación corporal, ansiedad físico social, desórdenes de alimentación) que el proceso de internalización de un cuerpo ideal produce en la persona es tomar conciencia del hecho de que estos ideales de belleza promovidos socialmente son inalcanzables. Precisamente, esta imposibilidad de alcanzar un ideal que no es real es lo que garantiza que su persecución lleve respuestas cognitivas, afectivas y conductuales negativas para la salud de la persona (CARRARD; ROTHEN, RODGERS, 2020). No obstante, dado que los estándares de belleza y delgadez son más rígidos para las mujeres que para los hombres (ZUECK et al., 2016), y sufren mayor presión social sobre la imagen ideal difundida por los medios (GRANDE-LÓPEZ, 2019), es lógico entender que este grupo poblacional sufra en mayor grado las consecuencias negativas asociados a la imagen corporal. De hecho, como muestran Sobrino-Bazaga y Rabito-Alcón (2018) las mujeres se muestran más autocríticas, se sienten más insatisfechas y culpables por no cumplir con los estándares estéticos que los hombres.

La presión hacia un ideal delgado se produce en torno a un discurso que defiende la búsqueda de la superación personal, la felicidad y el éxito individual (RODGERS, 2016). Aunque esta apariencia es poco realista, el discurso social defiende que todos podrían lograrla mediante el esfuerzo personal y el consumo de servicios y productos especializados. El continuo y permanente intento por alcanzar un ideal, que se muestra como común pero que es por sí inalcanzable, es lo que contribuye a un proceso normalización y a la formación del sujeto neoliberal.

\subsection{EL IDEAL DE CUERPO DELGADO Y LA FORMACIÓN DEL SUJETO NEOLIBERAL}

El discurso del ideal delgado proyecta dos claros elementos ideológicos del neoliberalismo (REVELEY, 2016). En primer lugar, supone una presión para lograr el ideal de delgadez que incita al consumo asociado con la industria de la belleza y del fitness. La cultura del consumo ha expandido la lógica del mercado a todos los ámbitos sociales y privados, de modo que las conductas sociales se explican en 
términos de actos de consumo, a través de los cuales el ser humano puede crear y recrear identidades mediante el disfrute de bienes y servicios, tanto en su uso material como simbólico (FEATHERSTONE, 1991). El cuerpo y la estética forman parte del sistema racional de producción de mercancías, y se han convertido en objetos de consumo por excelencia (GUTHMAN, 2009). El discurso del ideal delgado contribuye a la expansión del llamado culto al cuerpo. Se trata de un fenómeno multifacético y complejo, donde confluyen reclamos de valores como la salud, la estética o el placer, en torno a una imagen del cuerpo ideológicamente neutral, que impele al consumo y a la transformación corporal (VICENTE-PEDRAZ, 2010). No obstante, la realidad es que esta presión social antepone la ganancia económica a la salud de la persona (GARD; WRIGHT, 2005).

En segundo lugar, el discurso del ideal delgado se alinea con la búsqueda de la autonomía personal, la autosuficiencia y la responsabilidad por el propio bienestar. Se trata de un énfasis en la responsabilidad personal y la ciudadanía, pues acercarse al ideal delgado no sólo busca un beneficio personal, sino colectivo, dado que no hacerlo supone una carga y perjuicio para todos (GARD; WRIGHT, 2005; RODGERS, 2016). En línea con el discurso neoliberal, el ser humano es el artífice y responsable de acercarse al modelo corporal de referencia, una responsabilidad no sólo hacia uno mismo, sino también hacia la sociedad. Por lo tanto, la ideología neoliberal requiere individuos que han internalizado las normas del mercado y la autorresponsabilidad sobre su vida, un tipo de sujeto susceptible a los impulsos neoliberales (GUTHMAN, 2009).

La internalización del ideal, junto con las decisiones y comportamientos dirigidos hacia la consecución del cuerpo delgado, influyen en la construcción y en el reconocimiento de sí mismo como sujeto. Para esclarecer cómo estos procesos reflexivos dialécticos en relación con el discurso del ideal de cuerpo delgado pueden actuar y formar subjetividades, pueden ser especialmente útiles algunos de los presupuestos teóricos de Foucault.

El trabajo de Foucault se centra en los modos de subjetivación que transforman a los seres humanos en sujetos, a través del nexo que se establece entre el poder, la verdad y el yo (MARKULA, 2003). El concepto de sujeto, aquí, integra un doble significado: por un lado, que el individuo está "sujeto" a otro/s por el control y la dependencia, y, por otro, que está ligado a su propia identidad por una consciencia o conocimiento de sí mismo (FOUCAULT, 1994). Por su parte, el poder no se reduce a una esfera concreta, sino que es algo difundido en toda la sociedad, y tiene que ver con el gobierno de la conducta. Las relaciones humanas son todas relaciones de poder, una manera de influir unos en los otros que puede devenir en dominación o liberación (FOUCAULT, 1992).

Para Foucault (1992) no hay una verdad última de las cosas, sino que se construye mediante la producción de discursos que son reconocidos cómo válidos. En otras palabras, no existe una verdad, sino diferentes regímenes de verdad que compiten entre sí. La verdad, pues, está ligada a los sistemas de poder que la producen y mantienen, y a su vez esa verdad tiene efectos de poder que induce y reproduce, configurando un régimen de verdad. En la sociedad moderna, la verdad emana del 
conocimiento científico y de las instituciones que lo producen, creando un discurso sobre el ser humano que convierte el cuerpo en un espacio de normalización, toda vez que se legitima una visión del individuo como un objeto que puede ser medido, operativizado y clasificado.

Así, la hegemonía del discurso científico, particularmente la medicina (FOUCAULT, 2001), tiene efectos de dominación sobre el cuerpo, creando una sociedad disciplinaria donde el individuo es gobernado a través de sistemas de control de las operaciones del cuerpo (la vigilancia, la inspección y la normalización, fundamentalmente), que asegura un sometimiento constante e impone una relación de docilidad-utilidad (FOUCAULT, 2002). Se trata de una extensión del poder sobre la vida humana, acuñado por Foucault (1998) con el término biopoder, que presenta una doble vertiente: la anatomopolítica, que refiere a las formas de educar y disciplinar el cuerpo individual, y la biopolítica, que remite a las formas de administración de la vida de una población. De este modo, el biopoder ha sido un elemento indispensable para el desarrollo del capitalismo (FOUCAULT, 1998).

Ahora bien, una práctica discursiva particular puede ser moldeada por tecnologías de poder como medio de dominación, y por tecnologías del yo como medio de transformación personal (MARKULA, 2003). En este sentido, las tecnologías del yo "permiten a los individuos efectuar [...] operaciones sobre su cuerpo y su alma, pensamientos, conducta [...] obteniendo así una transformación de sí mismos con el fin de alcanzar cierto estado de felicidad, pureza, sabiduría o inmortalidad" (FOUCAULT, 1990, p. 48). Así, las tecnologías de poder dan lugar a proceso de objetivación, en contraste con las tecnologías del yo que dan lugar a un proceso de subjetivación, de modo que la constitución del sujeto es una relación dialéctica entre técnicas de dominación y técnicas del yo (FOUCAULT, 1990).

El discurso y la imagen corporal del ideal delgado podrían considerarse como una tecnología de poder en la medida en que "determinan la conducta de los individuos, los someten a cierto tipo de fines o de dominación, y consisten en una objetivación del sujeto" (FOUCAULT, 1990, p. 48). La imagen del cuerpo cosificado que promueven los medios sociales, señala ciertos ideales de sujetos a los que las personas debemos aspirar (DRAKE; RADFORD, 2019). La ideología neoliberal, además de habernos convertido en consumidores del cuerpo y la apariencia, alienta a las personas a pensar en sí mismas como individuos emprendedores (FOUCAULT, 2007). Así, estos mensajes sobre el ideal de cuerpo influyen en las personas y pueden orientar sus actuaciones sobre sí mismas, a través de las tecnologías del yo.

Si bien Foucault encuentra tecnologías del yo en las prácticas de liberación más que en las de dominación (McNAY, 1992), remarca que tales libertades están condicionadas por el contexto sociocultural en el que operan (HALL, 1996). Así, al sentirse vigilado, el individuo termina por auto-regularse, no tanto por iniciativa propia, sino por miedo a las consecuencias que puede acarrear en su cuerpo y su alma el desviarse del código (FOUCAULT, 2002). Esto podría llevar a los individuos a actuar en línea con la ideología dominante, convirtiéndose en formas de autocontrol y auto vigilancia. Como consecuencia, las imágenes y discursos sobre el cuerpo ideal delgado, podrían alzarse como referencias no sólo estéticas sino morales, en 
la medida en que el trabajo personal sobre el cuerpo en dirección hacia esos ideales es considerado una acción virtuosa (VICENTE-PEDRAZ, 2010). Así, tener un cuerpo delgado y atlético se asociaría hoy día al bienestar y al éxito, mientras que engordar representa la "metáfora del fracaso" (ESTEBAN, 2004, p. 83).

Siguiendo a Foucault (2007), la identidad neoliberal es la de un sujeto emprendedor de sí mismo, autónomo y responsable de su bienestar, dentro del marco de las condiciones económicas y políticas, y de las narrativas culturales de la sociedad contemporánea. Las personas, pues, son sujetos activos que se construyen a través de las tecnologías y las prácticas del yo (FOUCAULT, 1990). Si bien las tecnologías son mecanismos amplios, las prácticas del yo surgen en el nexo de acciones y discursos a medida que el poder se materializa en ciertas formas de pensar, hablar y comportarse (HARDY; THOMAS, 2015). Así, las personas construyen su subjetividad a través del pensamiento reflexivo, y sólo a través de la reflexividad como proceso psicológico del individuo, podemos entender cómo la gente actúa y cómo sus acciones están mediatizadas por las influencias sociales (HOUGHTON, 2019).

En síntesis, la imagen corporal y los discursos del ideal delgado actuarían como tecnologías de poder que contribuyen a la formación de sujetos neoliberales, trabajando en dos líneas. Por un lado, el ideal de cuerpo delgado favorece la insatisfacción ante la discrepancia cuerpo real-ideal (o normativo), lo que estimula el consumo a través de la transformación corporal. Por otro lado, los discursos del ideal delgado establecen referentes de éxito que han sido internalizados y enfatizan la responsabilidad individual por alcanzarlos. De este modo, el ideal inalcanzable de un cuerpo delgado funciona como una tecnología del yo en línea con la ideología neoliberal, dado que despierta en las personas procesos reflexivos de autocontrol y autoconstitución hacia el ideal normalizado que ha sido internalizado.

Aunque las personas pueden tener un nivel de elección sobre la forma en que se construyen a sí mismas a través del pensamiento reflexivo, sus acciones y valoraciones están influenciadas en mayor o menor grado por el contexto sociocultural. Por ello, es de interés para la investigación conocer cómo una persona se convierte en cierto tipo de sujeto en torno a los regímenes de verdad que se establecen sobre el cuerpo (HOUGHTON, 2019).

\subsection{EL PRESENTE ESTUDIO}

El objetivo de este trabajo es mostrar, a través de un caso, cómo las imágenes y discursos sobre el cuerpo ideal delgado pueden configurarse como dispositivos de poder que estimulan la formación de sujetos sensibles a la ideología neoliberal. Específicamente, tratamos de evidenciar cómo los referentes sobre el ideal de cuerpo delgado pueden funcionar como tecnologías de poder. Estas tecnologías de poder operan estimulando en la persona un proceso reflexivo de comparación entre la propia imagen corporal y la idealizada, que suele generar discrepancia e insatisfacción con el propio cuerpo. Esta insatisfacción fruto del proceso de normalización conduce a los individuos a seguir prácticas del yo, es decir, consumos del cuerpo (i.e., alimentación, ejercicio), al objeto de alcanzar esa norma que representa la imagen corporal ideal. De 
este modo, la persona se constituye en un sujeto "sujetado" a la influencia social sobre el ideal del cuerpo, un proceso que contribuye a formar subjetividades neoliberales. No obstante, es, precisamente, al entender cómo el individuo se constituye a sí mismo en sujeto en torno a los regímenes de verdad del ideal corporal, donde cabe la posibilidad de establecer otras prácticas. En este sentido, el trabajo finaliza delineando algunas implicaciones pedagógicas que puedan orientar las tecnologías del yo como prácticas liberadoras y de empoderamiento del individuo.

El estudio de caso es utilizado en este trabajo como una estrategia en la que el caso es seleccionado como reflejo de supuestos teóricos. Por lo tanto, no supone una representatividad estadística, sino que aporta representatividad sustantiva y transferibilidad sociológica respecto al objeto de investigación (CORBETTA, 2003). Por otro lado, el uso del caso único es utilizado con carácter crítico, en el sentido en que nos permite "confirmar, cambiar, modificar o ampliar el conocimiento sobre el objeto de estudio, lo que puede ser un factor importante para la construcción teórica” (LÓPEZ, 2013, p. 141).

En este sentido, el interés de esta investigación no radica en establecer relaciones empíricas causales ni generalizaciones, sino en aportar luz sobre la manera en que las personas interactúan dialécticamente con el contexto sociocultural en la construcción de su identidad, como muestran otros estudios de un solo caso (v. DEVIS; SPARKES, 1999; SICILIA; FERNÁNDEZ-BALBOA; ORTA, 2017). Por tanto, consideramos que este caso aporta conocimiento para ayudarnos a esclarecer los procesos psicosociales de constitución del sujeto neoliberal, de acuerdo con el objetivo formulado.

\section{MÉTODO}

Afrontamos este estudio de caso desde el paradigma naturalista siguiendo un enfoque narrativo crítico. En esta línea, nuestro interés es utilizar el relato de vida de una mujer, Adriana (seudónimo), para mostrar evidencias de los procesos de subjetivación neoliberal. Por lo tanto, es preciso aclarar que en este artículo no analizamos el relato al completo de Adriana, sino que utilizamos su historia como reflejo de la teoría, con la intención de aportar luz a los mecanismos de creación de subjetividades sensibles a la ideología neoliberal.

Los relatos y narraciones son formas de construir y representar identidades, y nos permiten comprender las subjetividades en relación con las condiciones socioculturales (DEVIS, 2017). La investigación narrativa se basa en cómo las personas dan sentido a su vida a través de relatos que ordenan sus vivencias y orientan sus acciones (MARTOS-GARCÍA; MONFORTE, 2019). Por tanto, los relatos de vida son personales, pero también sociales, pues cobran sentido en el contexto sociocultural en el que los vivimos, y permiten comprender fenómenos sociales más amplios a partir de la historia particular.

Cuando iniciamos este trabajo, Adriana era una estudiante de posgrado de 24 años de edad, graduada en Educación Primaria. La oportunidad de desarrollar esta investigación surgió, inicialmente, de manera espontánea durante una conversación 
informal sobre la moda, la apariencia y el cuerpo. Adriana se expresó abiertamente de una manera muy crítica hacia los estereotipos corporales del ideal delgado, mostrando incluso enfado en algunos momentos. Asimismo, confesó que sentía bastante malestar con su propio cuerpo, y cierto nerviosismo cada vez que se exponía socialmente. Esto nos sorprendió pues, desde nuestro criterio, la imagen corporal de Adriana era delgada e incluso tonificada, muy en línea con el ideal de belleza dominante.

Sin embargo, Adriana nos dijo que se sentía insegura con su cuerpo desde niña, pues entonces estaba gorda y ella deseaba estar delgada. Esta discrepancia le hizo sufrir mucho durante su etapa escolar. En Educación Primaria sufrió acoso escolar por su gordura, y su insatisfacción corporal desembocó en un trastorno de la alimentación en Secundaria que, según Adriana, dejó una huella permanente: un mecanismo psicológico de valoración y comparación continua de su cuerpo con el ideal delgado, y de autovigilancia y autocontrol respecto a la comida y la actividad física.

Los investigadores nos encontrábamos inmersos en el análisis crítico sobre la imagen corporal en las sociedades neoliberales. Así, identificamos su caso como una oportunidad para analizar empíricamente los procesos de construcción de los sujetos en la sociedad moderna contemporánea, a partir de las narrativas sobre el cuerpo y la apariencia. A pesar de su corta edad, lo que Adriana contó en este primer encuentro nos resultó muy interesante, pues reflejaba una gran riqueza de episodios vitales. Pero, además de ello, nos interesó en particular observar que Adriana concedía mucha importancia a su imagen corporal y constituía un factor relevante de su identidad. Por todo ello, le propusimos llevar a cabo la investigación, que aceptó inmediatamente, y procedimos al diseño de la misma.

Adriana fue informada de los objetivos, método, procedimiento, y los potenciales riesgos y beneficios de su participación en el estudio. Asimismo, le informamos de nuestra intención de difundir un informe del trabajo, garantizando en todo momento su anonimato y la confidencialidad de sus datos personales. Adriana estuvo de acuerdo y firmó un consentimiento informado.

El instrumento utilizado para la recogida de datos fue la entrevista cualitativa. Realizamos un total de 4 entrevistas, entre los años 2017 y 2019. Las tres primeras entrevistas fueron abiertas, orientadas a la elaboración de un relato que reconstruyera las sucesivas etapas de la vida de Adriana, al tiempo que nos permitieron identificar los principales temas emergentes. Una vez seleccionadas las unidades temáticas y las categorías relacionadas con el objetivo de esta investigación, diseñamos una última entrevista semiestructurada para profundizar y aclarar algunas de las ideas necesarias para la elaboración del presente artículo.

El lugar de las entrevistas fue cómodo e íntimo, elegido por la propia Adriana, y la manera como se desarrollaron nuestras conversaciones fue fluida favoreciendo un clima de confianza adecuado para poder tratar en profundidad los asuntos objeto de investigación. Todas las entrevistas fueron grabadas en soporte digital y transcritas a través de un procesador de textos. 
El análisis de los datos se centró en el análisis categorial de contenido (PÉREZ; FUENTES; DEVIS, 2011). De acuerdo con Polkinghorne (1995), hay dos maneras hacerlo: observando inductivamente los temas que surgen y los conceptos que se desarrollan en las historias; o utilizando los conceptos que emanan de la teoría para observar el reflejo de los mismos dentro de los datos. En esta investigación aplicamos ambas formas de análisis en dos fases sucesivas.

La información recogida en las tres primeras entrevistas fue sometida a un análisis de contenido categorial, identificando inductivamente las siguientes categorías emergentes: obesidad infantil, internalización ideal delgado, discrepancia en la imagen corporal, insatisfacción corporal, acoso escolar, ansiedad físico-social, rechazo social, trastornos de la alimentación, estereotipos corporales, diferencias de género, influencia medios de comunicación y redes sociales, presión social percibida, privación comida, ejercicio físico, autorreflexividad.

En este punto, el material del que disponíamos era rico y extenso, con temáticas diferentes que permitían distintos enfoques de investigación. Al discutir sobre dichas posibilidades en función de los presupuestos teóricos, los investigadores decidimos centrarnos en aquellas categorías que nos permitieran evidenciar el proceso de subjetivación vivido por Adriana, a partir de la relación con su cuerpo y la influencia de la imagen corporal ideal promovida por el discurso del ideal delgado. Así, temáticas como el género, los trastornos de la alimentación o el acoso escolar no fueron contempladas como categorías analíticas específicas, sino como elementos de la historia particular de Adriana que interactúan en el proceso de subjetivación neoliberal, dentro del marco teórico establecido.

Del contraste con la teoría y de acuerdo con el objetivo de la investigación, definimos tres nuevas categorías más amplias, que fueron utilizadas para, deductivamente, observar su reflejo en los datos obtenidos en la última entrevista y como eje de la discusión de este trabajo, a saber:

La imagen corporal ideal como tecnología de poder: ideal corporal delgado, discursos en medios sociales, manifestaciones de Adriana respecto al discurso del ideal delgado, presión social percibida.

Autorreflexividad, discrepancia e insatisfacción corporal: procesos reflexivos de Adriana sobre su cuerpo, sentimiento de discrepancia entre su cuerpo y el ideal delgado, manifestaciones de malestar con su imagen.

Prácticas del yo y construcción de subjetividades neoliberales: estrategias para la disminución o el control del peso, dieta, privación de alimentación, ejercicio físico, autorresponsabilidad, autovigilancia, identidad.

Para garantizar la calidad y el rigor de la investigación, realizamos un proceso de triangulación múltiple. En primer lugar, a través de un proceso de corroboración teórica, los investigadores discutimos extensamente sobre las conexiones del caso de Adriana con diversos argumentos teóricos críticos. En segundo lugar, un informe previo al presente fue analizado por un investigador ajeno, actuando como amigo crítico (SPARKES; SMITH, 2014), lo que estimuló la discusión teórica y metodológica. Finalmente, también seguimos un proceso de triangulación con Adriana, haciéndole 
llegar los sucesivos informes de la investigación hasta llegar al presente artículo. Este consentimiento dinámico durante el proceso (SPARKES; SMITH, 2014), permitió que Adriana conociera la evolución y el uso de los datos procedentes de su relato, y aportó mayor rigor a la investigación, así como un modo de hacerla más democrático y participativo (MARTOS-GARCÍA; MONFORTE, 2019).

\section{RESULTADOS Y DISCUSIÓN}

\subsection{LA INTERNALIZACIÓN DEL IDEAL DE CUERPO DELGADO Y CONSECUENCIAS ASOCIADAS}

De acuerdo con Foucault (2007), cuando estudiamos la influencia de una sociedad sobre los individuos y sus conductas, es importante focalizar la atención sobre qué clase de cuerpo se necesita, sobre qué cuerpo se promueve. El discurso del ideal delgado representa una imagen del cuerpo, que se fomenta socialmente de una manera tan generalizada que los niños comienzan a escuchar y a internalizar sus mensajes desde edades muy tempranas, alrededor de los cinco o seis años (KNAFO, 2016). Tal es el caso de Adriana:

Cuando era muy pequeña, recuerdo ir a la farmacia con mi madre, y la chica era super guapa, super alta y super delgada. Yo era chiquitita, rechonchita, todo lo contrario, y yo decía de mayor quiero ser así, delgada como ella.

Los mensajes de los medios sociales conllevan una presión que facilita la internalización del ideal delgado (RODGERS, 2016), a través de una constante comparación y monitorización del cuerpo. El cuerpo se valora principalmente en un sentido estético, de tal modo que se contrasta la propia imagen con los prototipos de belleza ideales (RAMOS et al., 2019). Se activa, pues, un proceso reflexivo que evalúa la imagen corporal real respecto a la ideal, y contribuye a la construcción de subjetividades condicionadas por el contexto sociocultural (HOUGHTON, 2019):

Miraba mi cuerpo y el de la chica [de la farmacia] y pensaba, yo quiero ser delgada. Tenía clarísimo que quería tener un cuerpo delgado y que eso era ahora temporal, porque era pequeña y tenía que dar un estirón, y los quilos se irían. Ese era mi pensamiento.

Esta regulación de lo corporal tiene importantes consecuencias en la vida de las personas: nos valoramos a través de la apariencia y los cambios corporales, convirtiendo una oportunidad de liberación y autodeterminación, en un instrumento de control que nos lleva a compararnos continuamente unas con otras (ESTEBAN, 2004). Esto hace emerger una discrepancia entre el cuerpo real y el ideal, que puede ser constatada a través de la comparación de mi propio cuerpo con el de los demás:

Yo siempre desde pequeña, al ser una chica gordita, pues me sentía un poco infravalorada. Me sentía mal conmigo misma, me comparaba muchísimo con mis compañeras porque eran delgadas... el prototipo considerado ideal. Entonces, claro, yo me sentía siempre el patito feo [...] Tenía clarísimo que yo de mayor quería tener un cuerpo delgado.

La situación de Adriana se vio alterada cuando, aún en Primaria, los compañeros del colegio comenzaron a burlarse de ella debido a su sobrepeso y a excluirla: 
Sola, me sentía muy sola. Porque también es cierto que, a raíz de esos comentarios, las amigas que yo tenía en el colegio se dejaban guiar un poco y me dejaban de lado [...] en excursiones ir yo sola en el autobús, por ejemplo, sin compañeros al lado porque nadie quería venirse conmigo. Miles de cosas así.

La literatura ha destacado que, junto con los medios sociales, los mensajes del entorno, en especial familia e iguales, tienen un impacto poderoso en la internalización de los ideales de belleza durante la infancia (KNAFO, 2016). De hecho, en el caso de Adriana la presión no procedió tanto de la familia como de los compañeros del colegio: "Si no hubiera tenido a esas personas que me recalcaban que yo era diferente, que yo estaba gorda, a lo mejor no me hubiera obsesionado tanto".

El ideal de cuerpo delgado señala un prototipo de belleza normalizado, que excluye a aquellos cuya apariencia se aleja de los prototipos (VICENTE-PEDRAZ, 2010). Adriana vivió un doble proceso de rechazo y exclusión: por una parte, de su entorno escolar; y, por otra, el que se hacía a sí misma, un proceso de autocrítica que empezó a hacerse más intenso a raíz de este incidente:

Y me autojuzgaba, me decía que fea soy, nadie me quiere, qué mal me siento, qué gorda estoy. En primero de ESO estaba a gusto en clase, pero a mí ya se me había quedado por dentro eso que ya había vivido antes. Con la gente, aunque no me diga nada, yo sé que me ven gorda, que me ven fea, que me ven... ¿sabes?

La internalización del ideal de cuerpo delgado por parte de Adriana comenzó a producir lo que Foucault denomina autovigilancia. La autovigilancia es un claro reflejo de que la presión hacia un ideal de cuerpo pasa al interior, es decir, es internalizado por la persona. Así, la presión del exterior no es sólo la que fuerza a Adriana a ajustarse al ideal corporal, sino que comienza a ser ella misma quien ejerce presión para ajustarse al ideal de cuerpo.

Esta presión añadida no sólo desde su entorno más próximo, sino también por ella misma, aumentó el malestar con su cuerpo y Adriana empezó a rechazar ir al colegio. Las consecuencias negativas comenzaron a ser evidentes en Adriana. EI acoso escolar vivido comenzó a vislumbrarse en ansiedad e insatisfacción corporal que, como en otros casos, derivó en un trastorno de la conducta alimentaria (ESCANDÓN-NAGEL et al., 2019):

Llegué a segundo [de ESO], me cambié el chip y dije, ese año yo paso, no voy a estar otra vez gorda como una vaca, aquí siendo la vergüenza de la clase [...] Entonces mi pensamiento fue dejar de comer y ya está [...] Así, durante seis u ocho meses y perdí mogollón de kilos [...] había semanas que me comía al día tres manzanas.

El trabajo de Argyrides y Sivitanides (2017) evidencia que las personas con una mayor discrepancia entre su peso real e ideal tienen niveles más altos de ansiedad relacionada con el cuerpo, tienen una alimentación más desordenada y un mayor nivel de internalización de los ideales corporales. Esta discrepancia puede conducir a sentir insatisfacción corporal, y catalizar un proceso continuo de autocrítica (SOBRINO-BAZAGA; RABITO-ALCÓN, 2018), que tiene importantes consecuencias sobre el autoconcepto (PUTRI et al., 2019). 
De acuerdo con Argyrides y Sivitanides (2017), las niñas internalizan en mayor medida que los niños el ideal de delgadez y son más sensibles a las presiones sociales de los medios. Consecuentemente, tienen más probabilidad de experimentar insatisfacción corporal y un mayor deseo de adelgazar, lo que conlleva niveles más bajos de autoestima y confianza en sí mismas. Los problemas vinculados con la insatisfacción corporal pueden relacionarse con episodios de ansiedad físico social (AHADZADEH et al., 2018), siendo la adolescencia uno de los periodos más críticos, con altos índices de preocupación por el cuerpo, especialmente en las chicas (RAMOS et al., 2019).

\subsection{LA SUJECIÓN AL IDEAL DE CUERPO DELGADO}

El discurso del ideal delgado señala unos ideales de cuerpo y apariencia que suelen asociarse a la salud y, sin embargo, no pueden ofrecer salud en la medida en que no responden a la diversidad de los cuerpos (RODGERS, 2016). La fuerza del discurso del ideal delgado está precisamente en el hecho de que cuando se internaliza el sujeto renuncia a su cuerpo real en busca de un ideal inalcanzable:

\footnotetext{
Sinceramente pienso que no es una muestra real de lo que podría conseguir todo el mundo. Es que es algo que ni lo veo saludable, en algunos casos excesivos de delgadez en las modelos o de hormonas en los hombres para tener un cuerpo más fuerte y potente. Me parece aceptable lo que haga cada uno, pero también creo que cada persona tiene que ser qué menos que un poco original y no parecer una máquina que hace todo lo que la sociedad establece como correcto.
}

El extracto de la entrevista de Adriana apunta tres cuestiones importantes. La primera, que es consciente que el modelo corporal ideal delgado no refleja sus propias condiciones y posibilidades, su cuerpo real. La segunda cuestión apuntada por Adriada es que el ideal corporal proyecta una visión homogénea del cuerpo, considerado una máquina. Finalmente, Adriana destaca el carácter normativo y normalizador, pues, como referencia corporal, acercarse al ideal es alinearse con lo que la sociedad aprueba. Sin embargo, Adriana quería tener un cuerpo como el ideal delgado, creyendo que era su elección libre:

Cuando pesaba menos me sentía bien pero mal. Me sentía bien porque me veía y digo estoy perdiendo peso, estoy buscando el cuerpo que siempre he querido según mis criterios, ¿no? Pero luego me sentía mal porque digo, joder, me apetece comerme esto [...] A veces ya no sabía qué hacer [...] por mí comería de todo lo que me gusta [...] pero claro, luego no me gusta verme gorda y la gente... ya sabes, puede ser muy cruel.

El fragmento anterior muestra la ambivalencia propia de su proceso de subjetivación, consecuencia de la lucha interna de Adriana entre seguir sus propios criterios o los que la sociedad demanda, llegando a cuestionarse si realmente es libre. Se trata de una dialéctica de aceptación/rechazo de los imperativos de normalidad estética.

Pero, ¿a quién representa esa normalidad estética? El ideal de cuerpo delgado se presenta como uno de los posibles regímenes de verdad a través de los que se materializa la lucha de grupos. De acuerdo con Vicente-Pedraz (2010), el cuerpo es la objetivación más indiscutible del gusto de clase, por lo que esta imagen 
corporal modélica representa "los esquemas de verdad y de verdad práctica propios de la clase dominante" (p. 136). Se trata, pues, de "un dispositivo de normalización biopolítica [...] cuyo reclamo más persuasivo es la consecución del cuerpo modélico" (p. 135).

Un ejemplo de esto lo proporciona el análisis de Venturini et al. (2020) sobre la actividad en Instagram de las llamadas musas fitness. A través de discursos e imágenes, estas mujeres, iconos de la cultura fitness del cuerpo sano, expresan cómo construir sus cuerpos y difunden prácticas dirigidas a tal fin, que incluyen ejercicio físico, ropa, cosméticos, alimentos y accesorios. Muestran su estilo de vida exitoso, reafirmando la idea de que la felicidad es el resultado de la exhibición de sus cuerpos, cuya mejora brinda oportunidades para una vida mejor.

Este cuerpo de éxito social, aunque sea reconocido como excluyente y homogéneo, distante de las posibilidades corporales de la mayoría (RODGERS, 2016), está intensamente presente en la cotidianidad, en las mentes de los individuos, influyendo, de diversas maneras, en la forma en que se ven a sí mismos (HOUGHTON, 2019). Es decir, "constituye la expresión de una identidad social, no mayoritaria, que a menudo es naturalizada y, en tanto que tal, legitimada y aplicada universalmente" (VICENTE- PEDRAZ, 2010, p. 135). Además, el discurso del ideal delgado contribuye a la interiorización de la creencia en la propia capacidad y autorresponsabilidad para controlar por sí mismo el peso y la apariencia física (CARRARD; ROTHEN; RODGERS, 2020): "Sé que esos cuerpazos no son reales [...] Pero sí, las redes sociales influyen un montón [...] pero, también, para qué te voy a engañar [...] a veces creo que si me esfuerzo igual puedo tener un tipo así".

Como desvela Adriana, los medios sociales constituyen mecanismos de dominación que aportan orientaciones morales que regulan la relación con nuestros cuerpos (ESTEBAN, 2004). Como tecnologías de poder, condicionan las creencias, actitudes y conductas de las personas, tratadas, así, como puros objetos (FOUCAULT, 1990), y son referencias del estilo de vida de las clases dominantes (VICENTEPEDRAZ, 2010), con una gran capacidad de influencia.

En este sentido, el trabajo de Venegas-Ayala y González-Ramírez (2020) evidencia un importante efecto significativo de la publicidad sobre la percepción de la imagen corporal en las mujeres. No en vano, sobre las mujeres pesa aún más la rigidez de los estándares de belleza, la presión de los medios de comunicación (GRANDE-LÓPEZ, 2019) y esa continua observación y objetivación de sus cuerpos (ZUECK et al., 2016):

Pues sí, nos machacan mucho con el hecho de que la mujer tiene que tener unas medidas perfectas [...] también se lleva la chica fitness, ¿no? Chicas con un culo que no es ni normal, unas pedazo de protuberancias que parecen globos [...] Además, a las mujeres continuamente nos miran por la calle, nos dicen cosas sobre nuestro aspecto [...] sí la verdad es que yo me siento muy observada, analizada, no sé.

De acuerdo con Drake y Radford (2019), los medios contemporáneos siguen, en gran medida, abordando el cuerpo de las mujeres como un objeto público susceptible de ser sometido a juicio. Para ellos, los anuncios de fitness dirigidos a corredoras recreativas tratan el cuerpo como una máquina y establecen prescripciones que 
enseñan cómo es ese cuerpo ideal y cómo debe funcionar de manera adecuada; es decir, funcionan como una pedagogía coherente con la ideología dominante, una muestra de la proliferación de prácticas (ejercicio, dieta, cirugía, etc.) destinadas a modificar los cuerpos (VICENTE-PEDRAZ, 2010).

Así, las disposiciones biopolíticas dominantes se introducen en la consciencia de las personas de modo que, en cierto momento, ellas activan por sí mismas los mensajes exteriores, ponen en marcha procesos de autovigilancia, y siguen comportamientos y transformaciones de su cuerpo en línea con el régimen de verdad dominante (FOUCAULT, 1990). Estas prácticas del yo conllevan no sólo un cambio en la apariencia física, sino que inciden en los pensamientos y sentimientos sobre uno mismo (VENTURINI et al., 2020), al mismo tiempo que condicionan las decisiones y trayectorias de vida que elegimos. Adriana asumió estrategias para el control del peso en busca de una apariencia cercana a las referencias sociales, a través, principalmente, de dos prácticas en diferentes momentos de su vida: la alimentación y el ejercicio físico.

Durante la adolescencia Adriana decidió la privación de comida. Este trastorno alimentario, relacionado con una percepción negativa de su imagen corporal, le afectó de manera significativa a su autoestima (ESCANDÓN-NAGEL et al., 2019). Pero, además, activó un mecanismo psicológico de autovigilancia y autocrítica permanente:

Cuando tú sufres eso, no te llegas a curar del todo. Porque a ver yo hoy día sí como, pero no con la libertad que comía de niña. Ya es que vas contando calorías con el miedo ¿engordaré? [...] El miedo a volver... a ver yo a día de hoy no me siento feliz con mi cuerpo de decir joh dios mío!, no, para qué te voy a engañar, sigo teniendo mis cosillas de sentirme inferior y demás.

\subsection{LA PRODUCCIÓN DE SUBJETIVIDADES ALTERNATIVAS}

Como sugiere Markula (2003), cuando una persona adopta una práctica corporal puede ser consecuencia de un proceso de sumisión a las tecnologías del poder o un medio para la transformación a través de las tecnologías del yo. Las prácticas para el control de peso, alimentación y ejercicio físico fundamentalmente, pueden responder a esta dialéctica. El discurso del ideal delgado fue, para Adriana, el régimen de verdad casi exclusivo, lo que la llevó en la adolescencia a conductas extremas como la privación de la comida. Sin embargo, su preocupación por mantener el peso y la apariencia que deseaba, le hizo transitar hacia otras prácticas, concretamente, la alimentación sana y el ejercicio físico:

Ya cuando volví a comer no dejé de hacerlo [...] luego engordé otra vez y así estuve un tiempo, más gorda más flaca [...] pero al final me interesé por la alimentación sana, más equilibrada [...] y ya a partir de la universidad aprendí a comer bien [...] También empecé a hacer ejercicio físico y hasta ahora.

Estas nuevas prácticas responden al encuentro con un nuevo 'régimen de verdad', un nuevo ideal de cuerpo, en este caso el que representa la cultura fitness:

Hoy día te puedo asegurar que tengo otro, no trastorno, pero sí es verdad que lo he guiado un poco más al deporte [...] Estaba en pleno boom el cuerpo fitness, musculoso, fibrado... digo pues ya está al gimnasio [...] Sí es verdad, estoy un poco obsesionada [...] me da igual cómo me miran los 
demás, soy yo la que me juzgo. Soy mi propio demonio [...] Nunca voy a decir estoy satisfecha [...] eso no va a existir en mi mente en la vida [...] Creo que nunca dejaré de hacer ejercicio y de estar pendiente de la comida.

Actualmente, Adriana mantiene estrategias orientadas a mejorar su cuerpo, una muestra de cómo la tecnología del yo se modifica para adaptarse a la narrativa dominante del momento, a través de diferentes prácticas (FOUCAULT, 1990), pero que mantienen la dirección hacia la consecución del cuerpo ideal. De hecho, Adriana expresa claramente cómo la imagen corporal ideal se ha convertido para ella en normativa, pues reconoce una permanente insatisfacción con su cuerpo y se siente responsable de las acciones para mejorar su imagen.

Así, como plantea Foucault (1990), en las prácticas asociadas con las tecnologías del poder y del yo, se encuentran los medios por los cuales los individuos se autorregulan, se auto-fabrican y se auto-producen. Un proceso de construcción de sí mismo como un sujeto neoliberal que reproduce, a través de sus propias prácticas y discursos, la 'verdad' sobre el cuerpo (HOUGHTON, 2019).

La identidad neoliberal, de acuerdo con Hall (1996), se construye a través de la diferencia no fuera de ella, pues la descripción del sujeto neoliberal ideal necesita la implicación de otro sujeto neoliberal: el sujeto no ideal. En lo relativo al cuerpo, esta diferencia se evidencia confrontando la imagen ideal (junto con sus prácticas) y la imagen que está fuera de la norma: "la imagen hegemónica y a menudo privativa del cuerpo joven, delgado, terso [...] se opone y se diferencia de su contra-imagen, el cuerpo envejecido, grueso" (VICENTE-PEDRAZ, 2010, p. 132):

\footnotetext{
Porque yo misma soy también de guiarme mucho por la apariencia. Es triste, pero es así. Estamos en esta sociedad tan magnífica y maravillosa que la apariencia es lo primero que ves [...] Todos los días, mira ésta qué guapa, o en la tele mira ésta qué tipito tiene, no tiene ni celulitis ni estrías ni nada, iqué vida más maravillosa! [...] eso me hace pensar en mí y.... uff! Me veo fea, gorda... no sé.
}

Aquí, el cuerpo, más que un indicador de la identidad personal, funciona como un operador que clasifica y jerarquiza socialmente a las personas. Queda manifestada, pues, una cultura de cuidar el cuerpo que se convierte en un imperativo moral, pues estar fuera de los estándares de referencia se asocia a una falta de responsabilidad personal, de negligencia y, por tanto, es una elección que afecta al sujeto mismo (VENTURINI et al., 2020):

Intento ir todos los días al gimnasio [...] pero cuando no puedo ir me siento mal. No sé, como si estuviera fallando [...] Ya sé que es absurdo, pero así es mi cabeza $[. .$.$] no seas vaga, me digo [...] incluso he llegado a entrenar$ doliéndome la rodilla.

Para Lambert (2018), esta permanente disposición a cumplir con los ideales neoliberales, al confiar la construcción del yo a anclajes simbólicos inestables a expensas del compromiso, la comunidad y el bienestar social, posiciona a las personas como sujetos frágiles, precarios y "psicóticos":

\footnotetext{
Me juzgo a diario, eso yo soy totalmente consciente, y aparte también le pregunto a mi novio o a mi madre [...] Algo en mi mente no está del todo... amor propio tengo escaso y eso sí que es verdad importante [...] Yo me siento que estoy pero no estoy, y las redes sociales me influyen demasiado [...] Yo pienso que nunca me voy a sentir cómoda con mi cuerpo, es que yo creo que nadie.
} 
En conclusión, el relato de Adriana ha evidenciado cómo, para ella, el discurso del ideal delgado se convirtió desde muy pequeña en la referencia normativa respecto a su cuerpo. Este referente se ha mostrado como una tecnología de poder que Adriana internalizó y que le provocó una discrepancia psicológica y una permanente insatisfacción corporal. Amplificado por la presión social particular que vivió desde su infancia y adolescencia, el discurso del ideal delgado estimuló en Adriana el desarrollo de tecnologías del yo y prácticas encaminadas a la consecución de ese ideal, procesos psicosociales de autovigilancia y autocontrol que contribuyeron a desarrollar una subjetividad en línea con la ideología neoliberal. La evolución del cuerpo delgado al cuerpo fitness como referente normativo para Adriana, muestra la apertura de espacios para la negociación de la identidad en el marco de la dialéctica entre las tecnologías del yo y del poder.

Así pues, el neoliberalismo se ha mostrado como la forma de subjetivación dominante, basada en la estimulación del individuo emprendedor de sí mismo, pero dentro de las pautas y referentes normativos que estimulan la normalización. Si bien la forma cómo los individuos reflexionan dialécticamente con las influencias sociales es variable, en el caso de Adriana, la cultura del consumo neoliberal se ha evidenciado como una ideología dañina que despertó un nivel elevado de autocrítica y auto exigencia, como se ha observado en otros contextos (v. LAMBERT, 2018). Esta cultura fomentó la dependencia de prácticas cambiantes y transitorias en la formación del yo que, si bien podríamos considerar que en cierto sentido tuvieron un carácter liberador de transformación de sí misma (aprender a comer sano, ejercicio físico), se mantienen en línea con los preceptos del discurso normativo.

\section{REFLEXIONES FINALES E IMPLICACIONES PEDAGÓGICAS}

La combinación de la permanente insaciabilidad de nuestro deseo individual (producto de la discrepancia cuerpo real-ideal), junto con la autorresponsabilidad sobre nuestra imagen corporal y las acciones hacia la consecución del cuerpo aceptado (cuando no exigido), conforman un efectivo dispositivo de control neoliberal que incita al consumo a través del cambio corporal. Una ideología que, mientras nos incita y estimula a ser nosotros mismos, consigue que repitamos estándares y estereotipos, creyendo ser libres e independientes. Entonces, nuestra corporeidad se convierte en una mercancía económicamente rentable, y en un sutil y efectivo ejercicio de control social.

En este trabajo hemos ofrecido el caso de Adriana como un ejemplo para observar cómo las tecnologías del yo son utilizadas para la sujeción del sujeto, siendo la dominación la consecuencia más plausible. A través del proceso de subjetivación de Adriana en torno a los ideales del cuerpo delgado podríamos extraer algunas implicaciones para el campo de la educación. Como se ha mostrado, las prácticas del yo pueden aspirar a cumplir las normas sociales o a desafiarlas, pueden expresar formas de dominación o de resistencia. De acuerdo con Foucault (1990, p.24),

[. . .] el objetivo principal hoy no es descubrir, sino rechazar lo que somos. Nos es preciso imaginar y construir lo que podríamos ser para desembarazarnos de esta especie de "doble coerción" política que es la 
individualización y la totalización simultáneas de las estructuras del poder moderno [...] Nos es preciso promover nuevas formas de subjetividad, rechazando el tipo de individualidad que se nos ha impuesto.

Necesitamos, entonces, acciones que estimulen formas de construcción del yo que respeten la diversidad y estimulen el potencial personal, y sirvan de resistencia frente a la ideología neoliberal que propone un tipo de individuo homogéneo y normalizado. En este sentido, se podría proponer una doble acción pedagógicapolítica. Por un lado, los agentes sociales podrían reivindicar el apoyo a regímenes de verdad alternativos a los que promulgan los ideales de cuerpo, regímenes de verdad que reconozcan la diversidad de cuerpos y el respeto a esta diversidad. Los agentes sociales podrían presionar a las instituciones para que exijan normativas más claras que limiten determinados mensajes corporales que son irreales, tratando de reducir la presión que ejercen, especialmente sobre los más jóvenes.

Por otro lado, en una esfera más personal e individual, sería preciso apostar por estrategias pedagógicas que promuevan prácticas de libertad a partir de las tecnologías del yo. Ante el dominio de una moralidad basada en las leyes de la sociedad, Foucault (1994) sugiere una práctica ética basada en el cuidado de sí mismo, pues las prácticas de libertad requieren un uso responsable del poder en todas las situaciones cotidianas. Así, el cuidado de sí mismo, a través de la promoción de nuevos conocimientos, sería el primer paso hacia el cuidado de los demás (SICILIA; FERNÁNDEZ-BALBOA, 2009). No obstante, de acuerdo con Markula (2003), consideramos que la consciencia crítica de uno mismo es el aspecto más importante de la tecnología del yo en lo relativo a la imagen corporal. Sin una consciencia crítica, las prácticas de autocuidado no pueden convertirse en prácticas transformadoras de libertad. La actitud crítica, pues, debe conducir a una auto-construcción que refleje la resistencia del individuo contra la dominación.

Particularmente, la educación física tiene un importante potencial como medio para la vivencia y la reflexión en torno a la imagen corporal. Sin embargo, la visión técnica mecanicista que aún predomina, mantiene una visión del cuerpo-máquina ajena a las implicaciones éticas y políticas de lo corporal (VICENTE-PEDRAZ, 2013), que puede ser cómplice de la ideología neoliberal.

Necesitamos, entonces, un giro pedagógico hacia el desarrollo de la corporeidad, basado en procesos de toma de consciencia y autoconocimiento que respeten la diversidad de los cuerpos y ayuden al alumnado a aceptarse tal como es: una educación basada en el cuerpo que siente y es sentido, no sólo a través de la visión y de la imagen; en la integración de las experiencias intelectuales, emocionales, sociales y corporales; en dirigir la mirada hacia nuestro interior y, al mismo tiempo, mantener una vis crítica hacia los modelos sociales y sus mecanismos de poder. En definitiva, una educación que nos ayude a habitar nuestro cuerpo de una manera más libre, autónoma, responsable y equilibrada. 


\section{REFERENCIAS}

AHADZADEH, Ashfrat Sadat et al. Relationship between body mass index, body image, and fear of negative evaluation: Moderating role of self-esteem. Health Psychology Open, v. 5, n. 1, p. 1-8, 2018. Disponible en: https://journals.sagepub.com/doi/ full/10.1177/2055102918774251. Acceso: 15 ene. 2020.

ARGYRIDES, Marios; SIVITANIDES, Marisia. Body image, self-esteem, media, disordered eating and actual ideal weight discrepancy: Findings in Cyprus. The European Journal of Counselling Psychology, v. 6, n. 1, p. 63-74, 2017. Disponible en: https://ejcop.psychopen. eu/article/view/109. Acceso: 15 ene. 2020.

BORDO, Susan. Unbearable weight: Feminism, Western culture, and the body. Berkeley: University of California, 2003.

CAFRI, Guy, et al. The influence of sociocultural factors on body image: a meta-analysis. Clinical Psychology, v. 12, n. 4, p. 421-423, 2005. Disponible en: https://doi.org/10.1093/ clipsy.bpi053. Acceso: 15 ago. 2020.

CARRARD, Isabelle; ROTHEN, Stéphane; RODGERS, Rachel. Body image and disordered eating in older women: A Tripartite Sociocultural Model. Eating Behaviors, n. 38, p. 101412, 2020. Disponible en: https://doi.org/10.1016/j.eatbeh.2020.101412. Acceso: 20 ago. 2020.

CORBETTA, Piergiorgio. Metodología y técnicas de investigación social. Madrid: McGraw Hill, 2003.

DEVIS, José. La investigación narrativa en la educación física y el deporte. Movimento, v. 23, n. 1, p. 13-24, ene./mar. 2017. Disponible en: https://seer.ufrgs.br/Movimento/article/ view/71277/40954. Acceso: 12 set. 2018.

DEVIS, José; SPARKES, Andrew. Burning the book: a biographical study of a pedagogically inspired identitiy crisis in phyisical education. European Physical Education Review, v. 5, n. 2, p. 135-152, 1999. Disponible en: https://doi.org/10.1177\%2F1356336X990052005. Acceso: 10 jul. 2018.

DRAKE, Carly; RADFORD, Scott K. Here is a place for you/know your place: Critiquing "biopedagogy" embedded in images of the female body in fitness advertising. Journal of Consumer Culture, 2019. Disponible en: https://doi.org/10.1177/1469540519876009. Acceso: 20 ene. 2020.

ESCANDÓN-NAGEL, Neli et al. Imagen corporal en función del sexo y estado nutricional: asociación con la construcción del sí mismo y de los otros. Revista Mexicana de Trastornos Alimentarios, v. 10, n. 1, p. 32-41, 2019. Disponible en: http://www.scielo.org.mx/scielo. php?script=sci arttext\&pid=S2007-15232019000100032\&lng=es\&nrm=iso\&tlng=es. Acceso: 20 feb. 2020.

ESTEBAN, Mari Luz. Antropología del cuerpo. Género, itinerarios corporales, identidad y cambio. Barcelona: Bellaterra, 2004.

FEATHERSTONE, Mike. Cultura de consumo y posmodernismo. Buenos Aires: Amorrortu, 1991.

FOUCAULT, Michel. El nacimiento de la biopolítica. Buenos Aires: Fondo de Cultura Económica, 2007.

FOUCAULT, Michel. El nacimiento de la clínica. Buenos Aires: Siglo XXI, 2001. 
FOUCAULT, Michel. Hermenéutica del sujeto. Madrid: La Piqueta, 1994.

FOUCAULT, Michel. Historia de la sexualidad I. La voluntad de saber. Madrid: Siglo XXI, 1998.

FOUCAULT, Michel. Microfísica del poder. Madrid: La Piqueta, 1992.

FOUCAULT, Michel. Tecnologías del yo. Y otros textos afines. Barcelona: Paidós Ibérica, 1990.

FOUCAULT, Michel. Vigilar y castigar. Buenos Aires: Siglo XXI, 2002.

GARD, Michael; WRIGHT, Jan. The Obesity Epidemic: Science, Morality and Ideology. London: Routledge, 2005.

GRANDE-LÓPEZ, Victor. La hipersexualización femenina en los medios de comunicación como escaparate de belleza y éxito. Comunication Papers: Media Literacy \& Gender Studies, v. 8, n.16, p. 21-32, 2019. Disponible en: https://www.researchgate.net/ publication/334580876. Acceso: 10 feb. 2020.

GUTHMAN, Julie. Teaching the politics of obesity: insights into neoliberal embodiment and contemporary biopolitics. Antipode, v. 41, n. 5, p. 1110-1133, 2009. Disponible en: https:// onlinelibrary.wiley.com/doi/abs/10.1111/j.1467-8330.2009.00707.x Acceso: 20 ago. 2020.

HALL, Stuart. Introduction: Who needs identity? In: HALL, Stuart.; DU GAY, P. (eds.) Questions of cultural identity. London: SAGE, 1996.

HARDY, Cynthia; THOMAS, Robyn. Discourse in a material world. Journal of Management Studies, v. 52, n. 5, p. 679-696, 2015. Disponible en: https://onlinelibrary.wiley.com/doi/ full/10.1111/joms.12113. Acceso: 23 mar. 2019

HIGGINS, E. Tory. Self-Discrepancy: a theory relating self and affect. Psychological Review, v. 94, n. 3, p. 319-340, 1987. Disponible en: http://persweb.wabash.edu/facstaff/ hortonr/articles\%20for\%20class/Higgins.pdf. Acceso: 17 ene. 2019.

HOUGHTON, Elizabeth. Becoming a neoliberal subject. Ephemera: theory \& politics in organization, v. 19, n. 3, p. 615-626, 2019. Disponible en: http://www.ephemerajournal.org/ sites/default/files/pdfs/contribution/19-3houghton_0.pdf. Acceso: 14 feb. 2020.

KNAFO, Hannah. The development of body image in school-aged girls: a review of the literature from sociocultural, social learning theory, psychoanalytic, and attachment theory perspectives. The New School Psychology Bulletin, v. 13, n. 2, p. 1-16, 2016. Disponible en: http://www.nspb.net/index.php/nspb/article/view/270. Acceso: 2 sept. 2020.

LAMBERT, Aliette. Psychotic, acritical and precarious? A Lacanian exploration of the neoliberal consumer subject. Marketing Theory, v. 19, n. 3, p. 329-346, 2018. Disponible en: https://doi.org/10.1177/1470593118796704. Acceso: 14 feb. 2020.

LÓPEZ, Wilmer O. El estudio de casos: una vertiente para la investigación educativa. Educere, v. 17, n. 56, p. 139-144, 2013. Disponible en: https://www.redalyc.org/ pdf/356/35630150004.pdf. Acceso: 13 mar. 2019

MARKULA, Pirkko. The technologies of the self: sport, feminism and Foucault. Sociology of Sport Journal, v. 20, n. 2, p. 87-107, 2003. Disponible en: https://doi.org/ 10.1123/ ssj.20.2.87. Acceso: 2 sept. 2020.

MARTOS-GARCÍA, Daniel; MONFORTE, Javier. "Haz lo que puedas". Un estudio de caso sobre diversidad funcional y Educación Física. Ágora para la Educación Física y el Deporte, n. 21, p. 52-73, 2019. Disponible en: https://revistas.uva.es/index.php/agora/article/ view/4082. Acceso: 17 feb. 2020. 
McNAY, Lois. Foucault, and Feminism: Power, Gender and the Self. Cambridge: Polity Press, 1992.

PÉREZ, Victor; FUENTES, Jorge; DEVÍS, José. El análisis narrativo en la educación física y el deporte. Movimento, v. 17, n. 4, p. 11-42, oct./dic. 2011. Disponible en: https://doi. org/10.22456/1982-8918.24402. Acceso: 20 jun. 2018.

POLKINGHORNE, Donald E. Narrative configuration in qualitative analysis. In: HATCH, Amos; WISNIEWSKI, Richard. (eds.), Life History and Narrative. London: Falmer Press, 1995. P. 5-24.

PUTRI, Wulandari, et al. Gender and physical self-concept differences among college students. Advances in Social Science, Education and Humanities Research, v. 335, p. 211-217, 2019. Disponible en: https://download.atlantis-press.com/proceedings/ icesshum-19/125914643. Acceso: 15 ene. 2020.

RAMOS, Pilar, et al. The role of body image in internalizing mental health problems in Spanish adolescents: an analysis according to sex, age, and socioeconomic status. Frontiers in Psychology, v. 10, p. 1952, 2019. Disponible en: https://www.ncbi.nlm.nih.gov/ pmc/articles/PMC6714592/. Acceso: 15 ene. 2020.

REVELEY, James. Neoliberal meditations: How mindfulness training medicalizes education and responsabilizes young people. Policy Futures in Education, v. 14, n. 4, p. 497-511, 2016. Disponible en: http://dx.doi.org/10.1177/1478210316637972. Acceso: 20 mar. 2019

RODGERS, Rachel. The role of the "Healthy Weight" discourse in body image and eating concerns: An extension of sociocultural theory. Eating Behaviors, v. 22, p. 194-198, 2016. Disponible en: https://www.sciencedirect.com/science/article/abs/pii/S1471015316300848. Acceso: 20 ago. 2020.

SICILIA, Álvaro; FERNÁNDEZ-BALBOA, Juan Miguel. Reflecting on the moral bases of critical pedagogy in PETE: Toward a Foucaultian perspective on ethics and the care of self. Sport, Education \& Society, v. 14, n. 4, p. 443-463, 2009. Disponible en: https://doi. org/10.1080/13573320903217166. Acceso: 20 ago. 2020

SICILIA, Álvaro; FERNÁNDEZ-BALBOA, Juan Miguel; ORTA, Antonio. The structure and content of flow experience narratives in sport: The tale of an elite sportsman. Universitas Psychológica, v. 16, n. 1, p. 12-14, 2017. Disponible en: http://www.scielo.org.co/scielo. php?script=sci abstract\&pid=\$1657-92672017000100012. Acceso: 20 mar. 2020

SOBRINO-BAZAGA, Adriana; RABITO-ALCÓN, Mari Franzi. Diferencias de género existentes en la población general en relación a la insatisfacción corporal asociada al ideal de belleza: una revision sistemática. Estudios de psicología, v. 39, n. 2-3, p. 548-587, 2018. Disponible en: https://www.tandfonline.com/doi/abs/10.1080/02109395.2018.1517860 . Acceso: 17 oct. 2019.

SPARKES, Andrew C.; SMITH, Brett. Qualitative Research Methods in Sport, Exercise and Health. From process to product. Oxon: Routledge, 2014.

THOMPSON, J. Kevine, et al. Exacting beauty: Theory, assessment and treatment of body image disturbance. Washington, DC: American Psychological Association, 1999.

THOMPSON, J. Kevine; SCHAEFER, Lauren. Body image, obesity, and eating disorders. In: BROWNELL, Kelly. D.; WALSH, B. Timothy. (eds.), Eating disorders and obesity: a comprehensive handbook. New York, NY: The Guilford Press, 2018. p. 140-144. 
VENEGAS-AYALA, Karina S.; GONZÁLEZ-RAMÍREZ, Mónica T. Social influences in a model of body dissatisfaction, weight worry and bodily discomfort in Mexican women.

Acta Colombiana de Psicología, v. 23, n. 1, p. 7-17, 2020. Disponible en: http://www.doi. org/10.14718/ACP.2020.23.1.2. Acceso: 10 may. 2020.

VENTURINI, Ivana V., et al. Musas fitness e a tríade corpo-consumo-felicidade. Movimento, v. 26, e26003, 2020. Disponible en: https://doi.org/10.22456/1982-8918.86634. Acceso: 20 abr. 2020.

VICENTE-PEDRAZ, Miguel. Crítica de la educación física y Educación Física Crítica en España. Estado (crítico) de la cuestión. Movimento, v. 19, n. 1, p. 309-329, jan/mar 2013. Disponible en: https://doi.org/10.22456/1982-8918.35661. Acceso: 10 ab. 2018.

VICENTE-PEDRAZ, Miguel. La construcción social del cuerpo sano. El estilo de vida saludable y de las prácticas corporales de la forma como exclusión. Nómadas. Revista Crítica de Ciencias Sociales y Jurídicas, v. 28, n. 4, p. 129-145, 2010. Disponible en: https://www.academia.edu/10069278/. Acceso: 10 abr. 2018.

ZUECK, María del Carmen, et al. Imagen corporal en universitarios mexicanos: Diferencias entre hombres y mujeres. Retos: nuevas tendencias en educación física, deporte y recreación, n. 30, p. 177-176, 2016. Disponible en: https://recyt.fecyt.es/index.php/retos/ article/view/50217. Acceso: 19 mar. 2019. 
Resumo: O objetivo deste artigo é mostrar, através de um único estudo de caso, como a discrepância entre a imagem corporal do magro ideal e o real pode estimular os processos de formação do sujeito neoliberal. $O$ instrumento de coleta de informações foi a entrevista qualitativa, aberta e semiestruturada. Os resultados mostram como a imagem corporal do ideal magro pode funcionar como uma tecnologia de potência, exibindo um corpo ideal normalizado e homogêneo que estimula processos reflexivos críticos sobre si mesmo, processsos que podem levar a práticas de transformação do próprio corpo, contribuindo para a construção do corpo, contribuido para a construção de sujeitos sensíveis ao impulso neoliberal. Por fim, fornecemos algumas implicações pedagógicas para o desenvolvimento de tecnologias libertadoras de si voltadas para o autoconhecimento e o aumento da consciência crítica.

Palavras chave: imagem corporal. Padronização corporal. Relato de caso. Conscientização.

\begin{abstract}
As a single case study, this paper shows how the discrepancy between the thin-ideal body image and the real body can drive processes that form the neoliberal subject. Data were collected through qualitative, open and semistructured interviews. The results show how the thin-ideal body image can function as a power technology by exhibiting a normalized and homogeneous ideal body that stimulates critical reflective processes about oneself, which can lead to practices of transformation of the body itself, contributing to form subjects that are receptive to the neoliberal impulse. Finally, we point out some pedagogical implications for the development of liberating technologies of the self, aimed at self-knowledge and increasing critical awareness.
\end{abstract}

Keywords: Body image. Body patterning. Case reports. Awareness. 


\section{LICENCIA DE USO}

Este es un artículo publicado en Open Access bajo la licencia Creative Commons Atribución 4.0 Internacional (CC BY 4.0), que permite su uso, distribución y reproducción en cualquier medio, siempre que la obra original esté correctamente citada. Más información en: http://creativecommons.org/licenses/by-nc/4.0

\section{CONFLICTO DE INTERESES}

Los autores han declarado que no existe conflicto de intereses en este trabajo.

\section{CONTRIBUCIONES DE LOS AUTORES}

Cornelio Águila: fundamentación teórica, metodología, trabajo de campo, análisis, discusión, edición texto.

Álvaro Sicilia: fundamentación teórica, metodología, discusión.

Lidia Segovia: fundamentación teórica, metodología, trabajo de campo, análisis.

\section{FINANCIACIÓN}

El presente trabajo se llevó a cabo sin ningún apoyo financiero.

\section{CÓMO HACER REFERENCIA}

ÁGUILA, Cornelio; SICILIA, Álvaro; SEGOVIA, Lidia. El ideal de cuerpo delgado y la formación del sujeto neoliberal: un estudio de caso. Movimento, v.26, p. e26084, jan./dez. 2020. Disponível em: https://seer.ufrgs.br/Movimento/ article/view/104124. Acesso em: [día] [mes abreviado]. [año]. DOI: https://doi. org/10.22456/1982-8918.104124

\section{RESPONSABILIDAD EDITORIAL}

Alex Branco Fraga*, Elisandro Schultz Wittizorecki*, Ivone Job*, Mauro Myskiw*, Raquel da Silveira*

*Universidade Federal do Rio Grande do Sul, Escola de Educação Física, Fisioterapia e Dança, Porto Alegre, RS, Brasil 